\title{
Magnetic Resonance Imaging of Rectal Cancer
}

\author{
Ekta Maheshwari ${ }^{1}$ Gitanjali Bajaj ${ }^{1}$ Kedar Jambhekar ${ }^{1}$ Tarun Pandey ${ }^{1}$ Roopa Ram ${ }^{1}$
}

${ }^{1}$ Department of Radiology, University of Arkansas for Medical Sciences, Little Rock, Arkansas, United States

\begin{abstract}
Address for correspondence Roopa Ram, MD, Department of Radiology \# 556, University of Arkansas for Medical Sciences, 4301, West Markham Street, Little Rock, 72205, United States, (e-mail: RRam@uams.edu).

Ekta Maheshwari, MD, Department of Radiology \# 556, University of Arkansas for Medical Sciences, 4301, West Markham Street, Little Rock, 72205, AR, United States (e-mail: dr.maheshwariekta@gmail.com).
\end{abstract}

\begin{abstract}
Keywords

- magnetic resonance imaging

- rectal cancer

- preoperative staging

- treatment response

High-resolution magnetic resonance imaging (MRI) plays a pivotal role in the pretreatment assessment of primary rectal cancer. The success of this technique depends on obtaining good-quality high-resolution T2-weighted images of the primary tumor, orthogonal to rectal lumen. The goal of magnetic resonance staging is to identify patients who will benefit from neoadjuvant therapy prior to surgery to minimize postoperative recurrence and planning of optimal surgical approach. MRI also facilitates optimal identification of important prognostic factors, which improves both treatment selection and posttreatment follow-up. The objective of this article is to review the existing literature and provide a concise update on various aspects of rectal cancer imaging, discuss the current role of advanced imaging techniques such as diffusion-weighted and perfusion imaging in the evaluation of rectal cancer, and to assess response to therapy.
\end{abstract}

\section{Introduction}

Rectal cancer is one of the most common malignancies, with an incidence of 40 in 100,000. Colorectal cancer is the third leading cause of cancer-related mortality worldwide in both men and women. ${ }^{1}$ Adenocarcinomas comprise approximately $96 \%$ of all colorectal cancers, ${ }^{2}$ whereas the uncommon malignancies include lymphoma, gastrointestinal stromal tumors, and carcinoid. Rectal cancers are associated with poorer prognosis and higher local recurrence than their counterparts in the colon.

Although precise staging of rectal cancer is possible only with histopathology, magnetic resonance imaging (MRI) with its multiplanar imaging capacity and excellent soft tissue contrast plays a pivotal role in preoperative diagnosis and staging. MRI also facilitates optimal identification of important prognostic factors, which improves both treatment selection and posttreatment follow-up. One of the most important factors that govern the selection of treatment is the accuracy of rectal cancer staging, and high-resolution MRI is the mainstay of diagnosis for the same. MRI is now routinely used for evaluation of neoadjuvant therapy response, further guiding surgical approaches and oncologic treatment options. It is also an important guide to planning different surgical techniques where sphincter sparing and organ preservation is desired.

Total mesorectal excision (TME) is the standard of care, with additional neoadjuvant concurrent chemoradiation therapy (CCRT) in a select group of patients. In patients with locally advanced disease, neoadjuvant CCRT has improved local control and is associated with reduced toxicity when compared with postoperative adjuvant therapy. ${ }^{3}$ As such, initial staging investigations should identify the patients who benefit from preoperative therapy with intent to minimize the risk of postoperative tumor recurrence.

The purpose of this article is to review the existing literature and provide a concise update on various aspects of rectal cancer imaging, highlighting the controversies in areas such as anatomical staging, MRI technique, scan protocol, and pre- and posttreatment follow-up imaging. The article also reviews the current role of advanced imaging techniques such as diffusion-weighted and perfusion imaging in the evaluation of rectal cancer.

\subsection{5/s-0039-1683772}

License terms

()(1) $\Theta \circledast$ 


\section{Background}

Currently, surgical resection with stage appropriate neoadjuvant combined modality therapy is the mainstay of treatment of rectal cancer. In the past decade, the increasingly widespread adoption of TME has resulted in a dramatic decline in the prevalence of local recurrence from $38 \%$ to less than $10 \%{ }^{4} \mathrm{TME}$ is a surgical technique that entails resection of the entire rectal compartment and mesorectum by means of dissection along the planes of mesorectal fascia (MRF).

For tumors in the high rectum, low anterior resection (LAR) is the surgery of choice, whereby a portion of the distal rectum can be preserved. Tumors in the mid to low rectum are resected to the level of the pelvic floor muscles. For more distal tumors, sphincter-sparing surgeries, such as ultra LAR with coloanal anastomosis and intersphincteric resection with coloanal anastomosis, may be attempted. Utilization of a sphincter- sparing technique results in improved surgical outcome in terms of recurrence and postoperative complications and substantially improves quality of life. ${ }^{5}$ Abdominoperineal resection (APR) includes removal of the entire sphincter complex and is reserved for tumors in the low rectum that are fixed to adjacent pelvic organs or structures $^{6}$ ( - Figs. 1A, B, and C; and $\mathbf{2 A}$ and B).

"Circumferential resection margin" (CRM) is a pathologic term that refers to the surgically dissected surface of the specimen and corresponds to the nonperitonealized portion of the rectum. ${ }^{7}$ A positive CRM is defined as less than $1 \mathrm{~mm}$ distance between the surgical margin and tumor or malignant node and remains an important predisposing factor for local recurrence. ${ }^{8}$ This is an independent prognostic factor. ${ }^{9}$ Mesorectal fascia (MRF) is the extraperitoneal pelvic fascial plane that surrounds the mesorectum. MRF is visualized on MRI and is a more appropriate term for magnetic resonance reporting ${ }^{10,11}$ ( - Fig. 3). Peritoneal reflection is a thin T2 hypointense structure, best seen on sagittal or axial images, and reflects off the superior aspect of bladder onto the anterior aspect of rectum. Tumors that involve the reflection are categorized as T4a ( - Fig. 4).

\section{Imaging Modalities}

Preoperative staging, in particular, the assessment of extramural spread, has proven to be an important prognostic indicator
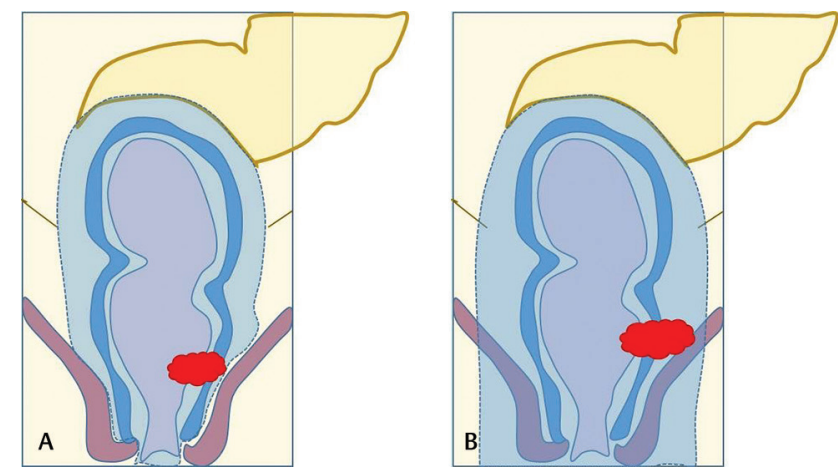

Fig. 2 Schematic representation of two types of abdominoperineal resection (APR) relative to position of the levator. (A) Intersphincteric APR. (B) Suprasphincteric APR.

in patients with rectal cancer. The primary modalities for local staging (T stage) in rectal cancer have been endoscopic ultrasound (EUS) and MRI, although previous studies have shown wide variability in the reported accuracy of both modalities (-Fig. 4). Over the past 10 years, the reported accuracy of EUS in the staging of rectal cancer has been highly variable, with values ranging anywhere from 69 to $89 \% .^{12,13}$ This variability is only partially due to improvement in ultrasonography over time and is instead more strongly a result of several other variables, including experience level of the sonographer, methodological differences between studies (e.g., small sample size, exclusion of occlusive cancers that prevent use of transrectal ultrasonography, and publication biases). ${ }^{14}$

Studies that directly compare EUS and MRI in staging of rectal cancer provide disparate results. One such metaanalysis showed that EUS is comparable to MRI for the evaluation of every $\mathrm{T}$ stage except in perirectal tissue invasion, in which EUS is significantly more sensitive. ${ }^{15}$ Conversely, another smaller study showed MRI to be slightly better at T-stage evaluation than EUS, with overall accuracies of 89.7\% and $85.2 \%$, respectively. ${ }^{13}$ Multiple studies have suggested that EUS is more sensitive than MRI for the assessment of T1 and T2 tumors, with no significant difference between the two in the staging of T3 and T4 tumors. Although this has implications for using EUS in the evaluation of superficial tumors, it is important to note that EUS is highly user-dependent, cannot assess stenosing tumors, and is not tolerated as well
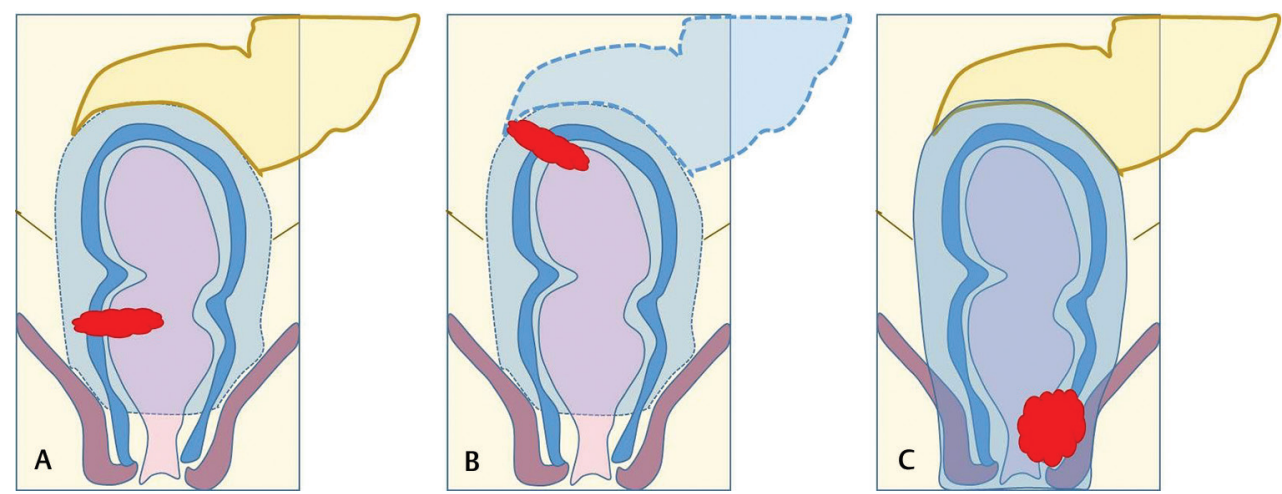

Fig. 1 (A-C) Schematic representation of different surgical approaches used for rectal cancer. (A) Total mesorectal excision. (B) Low anterior resection. (C) Abdominoperitoneal resection. 

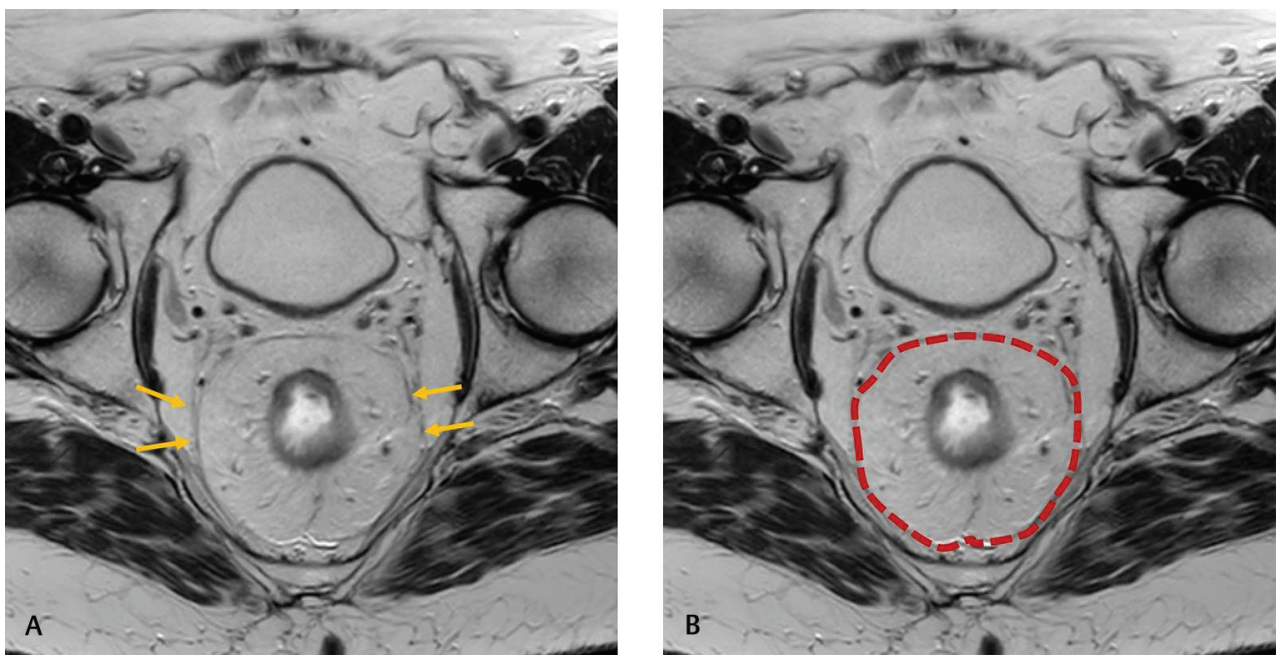

Fig. 3 (A, B) Margin of the mesorectal fascia with respect to which the circumferential resection margin is measured.
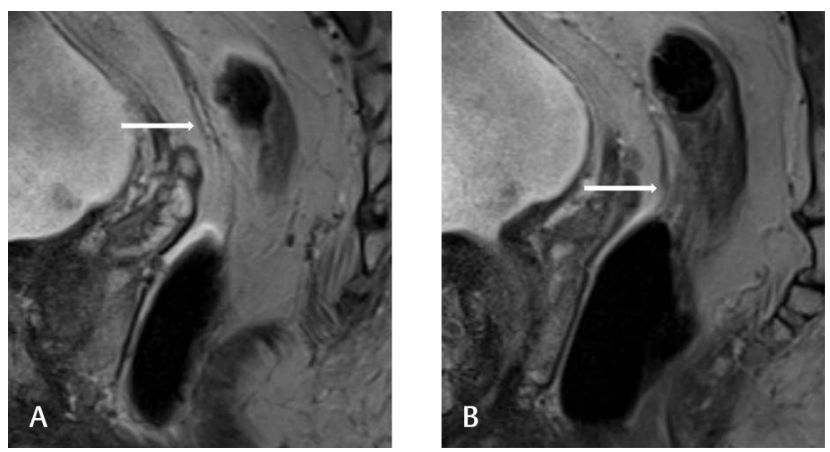

Fig. 4 (A, B) Peritoneal reflection (arrow).

as MRI. This, combined with the fact that extramural spread is the most important prognostic indicator with regard to $\mathrm{T}$ stage, tilts the balance toward MRI as the initial modality in assessing $\mathrm{T}$ stage, with the use of EUS only in supplemental assessment of superficial tumors. ${ }^{16}$

Although EUS is accurate for staging superficial rectal tumors, it has limited utility in the staging of more advanced disease, because the depth of acoustic penetration is generally unable to assess advanced-stage disease. ${ }^{12}$ It is also less suitable than MRI for evaluation of the mesorectal excision plane and mesorectal and extramesorectal lymph nodes. ${ }^{17}$ Owing to the excellent soft tissue contrast of MRI, limitations of operator dependence of EUS and inability to evaluate the fibrostenosing lesions. MRI plays a major role in staging, assessment of response to treatment and local recurrence.

Computed tomography (CT) is not recommended for local staging of rectal cancer, because it cannot reliably differentiate layers of the rectal wall, identify the MRF, or depict tumor invasion in surrounding pelvic structures..$^{15,18}$ It is essentially used to assess distant metastatic disease, such as liver and lung metastases.

Compiled results of studies on positron emission tomography (PET) in initial staging suggest that PET-CT provides additional information to conventional staging in primary rectal cancer. A study by Eglinton et al concluded that information from PET did not affect the surgical management and PET-CT may be most appropriately used selectively in more advanced stages and where indeterminate findings exist with conventional staging..$^{19}$ One of the recent reviews by Balyasnikova and Brown ${ }^{20}$ maintains that there is a paucity of literature with validated outcome data concerning use of PET-CT, hence their use in the initial assessment and restaging after treatment is limited to research protocols. Combination MRI and CT is essential for distant spread assessment and recurrent disease, and currently PET-CT is sometimes used in the workup of patients with recurrent and metastatic disease..$^{20}$

\section{Magnetic Resonance Imaging Protocol}

The optimal technique for MRI is somewhat variable according to the institution. An ideal technique should aim at acquisition of images that provide detailed assessment of layers of rectal wall, primary tumor, and its relationship to pelvic structures and assessment of regional lymph nodes.

\section{Pre-scan Workup and Patient Preparation}

Prior to MRI, part of the patient's workup, should include review of clinical and surgical history and prior imaging. The surgeon's notes regarding tumor location, circumferential extent, and distance from the anal verge should be reviewed. Use of spasmolytics to prevent artifacts from bowel motion has been recommended by some and glucagon is used at our institution. No specific bowel preparation is required. An empty bladder is preferable. Use of anterior saturation band is useful for reducing the artifact related to respiration.

Rectal distension for initial staging is a controversial point in the literature. As per Society of Abdominal Radiology Disease Focused Panel (SAR-DFP) recommendations and guidelines, rectal gel administration is not required for initial staging assessment. ${ }^{21}$ Although rectal distension can provide a more accurate assessment of the tumor stalk in polypoid masses, some studies have found that distension can actually alter the distance between rectal wall and MRF, potentially skewing measurements for the margin of resection. ${ }^{22}$ This is especially true in the lower rectum posteriorly where the fat plane between rectal wall and MRF is most susceptible to 
distension. Distension is most troublesome for T3 tumors that are the most likely to be upstaged, which can lead to unnecessary neoadjuvant therapy. ${ }^{17}$ Larger tumors or tumors in the lower rectum, on the other hand, should not be evaluated with rectal distension. ${ }^{23}$

At our institution the practice is to administer rectal gel for small tumors or for assessment following treatment when it is difficult to visualize the primary tumor. It has been our experience that tumors located near the rectosigmoid or in high rectum do not exhibit improved evaluation with rectal gel as sufficient distension is not achieved. In tumors such as these, there may be potential use for saline infusion concurrent with the use of a rectal balloon.

\section{Coils}

Endorectal coils provide high-resolution images that are able to delineate bowel wall layers. Some investigators have reported superior diagnostic accuracy compared with phased-array coils alone for T staging. ${ }^{24}$ Disadvantages of the endorectal coil include patient discomfort, increased cost of the coil, and increased examination time. Pelvic phased-array coils, with their increased field of view (FOV) and ability to image the entire pelvis, improved staging with better characterization of tumor invasion into perirectal tissue. Developments in phased-array coil technology have enabled high spatial resolution, high-contrast resolution scanning, which provides information comparable to that obtained with an endorectal coil. ${ }^{25}$ Endorectal coils are thus not used routinely in practice for rectal cancer staging. The current consensus is that pelvic phased-array coils provide the most detailed depiction of the rectal wall and surrounding structures. ${ }^{26}$

The coil should be positioned to cover rectum, mesorectum, and highest nodal drainage area, approximately $5 \mathrm{~cm}$ superior to the tumor. In general, this provides coverage from sacral promontory to below pubic symphysis. The lower edge of coil should be $10 \mathrm{~cm}$ below the pubic symphysis to acquire adequate signal from the low rectum and anus.

\section{Choice of Sequences and Scan Protocol}

An overview of the pulse sequences and their rationale based on the current evidence is summarized in - Table 1.

\section{T2-Weighted Sequence}

High-resolution T2-weighted images are the gold standard for evaluating rectal cancer. To help localize the primary tumor, an initial sagittal localizer sequence should be obtained from one pelvic sidewall to the other. This sequence serves as the baseline for planning high- resolution T2 axial images, orthogonal to the long axis of tumor, ideally performed with 3- to 4-mm slices using a small FOV ( - Fig. 5). Proper planning of high-resolution T2 imaging sequences is essential to staging accuracy. ${ }^{27}$ Not obtaining images perpendicular to the tumor axis may result in misinterpretation due to volume averaging, for example, blurring of muscularis propria or pseudospiculated appearance that could lead to overstaging ( - Fig. $5 \mathbf{A}$ and $\mathbf{5 B}$ ). ${ }^{23}$

Subsequently, large FOV coronal T2 sequences of the entire pelvis should be obtained. Coronal high-resolution T2-weighted images should be taken parallel to the long axis of the anal canal to evaluate sphincter involvement. Involvement of the peritoneal reflection and pelvic sidewalls is most clearly depicted on sagittal and coronal images, respectively. Triplanar imaging is of particular utility in the assessment of large or tortuous tumors where positioning of a single axial plane is difficult. Suzuki and colleagues have found that using a protocol including triplanar T2 sequences with highresolution imaging resulted in significantly better correlation with histopathology regarding anterior organ involvement. ${ }^{28}$

\section{T1-Weighted Sequence}

Turbo spin echo (TSE) T1W images are typically obtained in the axial plane at $3 \mathrm{~mm}$ to evaluate the mesorectal fat and
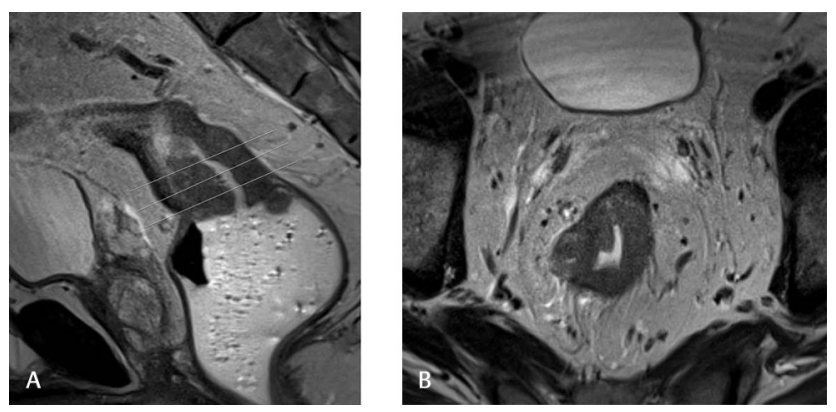

Fig. 5 (A, B) Planning of axial images using the sagittal image, in a plane orthogonal to long axis of the tumor.

Table 1 MRI protocol for rectal cancer imaging

\begin{tabular}{|l|l|l|l|l|l|l|}
\hline Sequence & Plane & $\begin{array}{l}\text { Echo time } \\
(\mathbf{m s})\end{array}$ & $\begin{array}{l}\text { Repetition } \\
\text { time }(\mathbf{m s})\end{array}$ & $\begin{array}{l}\text { Slice thickness } \\
(\mathbf{m m})\end{array}$ & Matrix & Comments \\
\hline High-resolution T2 & $\begin{array}{l}\text { Axial, coronal, } \\
\text { and sagittal }\end{array}$ & 80 & 1,250 & 3 & $320 \times 320$ & $\begin{array}{l}\text { Sagittal is performed first } \\
\text { to help plan }\end{array}$ \\
\hline $\begin{array}{l}\text { Diffusion-weighted } \\
\text { imaging }\end{array}$ & Axial & 60 & 1200 & 5 & $150 \times 150$ & $\begin{array}{l}\text { Tumor localization, post } \\
\text { treatment surveillance }\end{array}$ \\
\hline T1 & Axial & 500 & 10 & 3 & $256 \times 256$ & $\begin{array}{l}\text { Provides anatomic } \\
\text { information }\end{array}$ \\
\hline $\begin{array}{l}\text { 3D T2 iso-voxel } \\
\text { sequence }\end{array}$ & $\begin{array}{l}\text { Axial and } \\
\text { sagittal }\end{array}$ & 4,300 & 100 & 3 & $256 \times 256$ & $\begin{array}{l}\text { Can be reformatted into } \\
\text { any plane }\end{array}$ \\
\hline $\begin{array}{l}\text { 3D gradient echo } \\
\text { T1 }\end{array}$ & Axial & 1.1 & 3.2 & $\begin{array}{l}\text { Precontrast and multi- } \\
\text { phase postcontrast }\end{array}$ \\
\hline
\end{tabular}

Abbreviation: MRI, magnetic resonance imaging. 
lymph nodes. These are not helpful for bowel wall layer depiction. Though accuracy for staging between 1.5 -T versus 3-T magnets is almost similar, 3-T imaging offers the advantage of higher spatial resolution. ${ }^{26}$

\section{D Iso-voxel T2-Weighted Turbo Spin Echo Sequence}

At our institution, we routinely perform a 3D T2-weighted sequence, which can be reconstructed in any plane. This is performed if an ideal orthogonal sequence through the tumor has not been obtained. Three-dimensional images can be acquired in less time than two-dimensional (2D), and they provide similar accuracy in $\mathrm{T}$ and $\mathrm{N}$ staging. ${ }^{29}$ The only major limitation of 3D iso-voxel acquisition is sensitivity to patient motion.

\section{Intravenous Contrast}

As surmised from multiple studies, ${ }^{30-33}$ gadolinium contrast has not proved effective for rectal cancer staging and is not included in the recommended protocol by SAR disease focused panel. Jao and colleagues have found that gadoliniumenhanced T1-weighted MRI does not increase the diagnostic yield for tumor and nodal staging and can be omitted from the staging protocol. ${ }^{30}$ It has been proposed that intravenous contrast administration would help delineate areas of viable tumor within spiculations that are seen on both pre- and posttreatment images. However, studies have shown that the addition of gadolinium does not improve the radiologist's ability to determine accuracy of local invasion ${ }^{31}$ or posttreatment fibrosis and desmoplastic reaction versus tumor. ${ }^{32} \mathrm{~A}$ very recent review by Dijkhoff et al, however, shows that despite the large variability in DCE studies in rectal cancer, there seems a value for dynamic contrast-enhanced-MRI (DCE-MRI) in the staging and restaging of rectal cancer. ${ }^{34}$

Dynamic contrast imaging is, however, still a part of routine protocol at our institution. In our experience, it increases confidence levels for detection of small tumors, nodes, and incidental pelvic or bony abnormalities. It also adds value as the initial postcontrast study can serve as baseline while evaluating tumor response once the patient has received chemoradiation treatment (CRT). We advocate the use of dynamic postcontrast imaging, which is bolus triggered to capture arterial, early, and delayed venous phases, followed by axial and sagittal delayed images. Subtraction images can also be used to better evaluate the pathology of the tumor. Postcontrast images only add an extra 6 to 8 minutes to the scan time.

\section{Diffusion-Weighted Imaging}

Diffusion-weighted imaging (DWI) is now routinely performed for the evaluation of most tumors in the body. This free-breathing technique is based on free water diffusion, which is restricted in areas of high cellularity. At our institution, we use four b-values $(0,50,400$, and 800$)$ followed by a reconstructed apparent diffusion coefficient (ADC) map. Most solid tumors, including rectal tumors, show restricted diffusion on DWI. Low b-value images (50 and 400) provide maximal lesion detection, particularly for the presence of lymph nodes and bone metastases, whereas high b-value images (b value 800 ) provide signal suppression of highly cellular structures, such as gastrointestinal and urogenital lining, to maximize conspicuity of tumor. ${ }^{35}$ The higher signal intensity focus depicting a tissue with restricted diffusion is readily apparent against a low signal intensity background of bowel wall and feces on high b-value images. Hence, the sequence generally aids in detection of small tumors not seen on $\mathrm{T} 2$ images.

DWI is limited by its low spatial resolution as well as the fact that some benign tumors may also show restricted diffusion. For this reason, it has been suggested that evaluation of ADC may be more reliable, although some investigators think that ADC evaluation is cumbersome in practice. DWI is highly sensitive in nodal detection but has limited value for characterizing lymph nodes, because there is significant overlap in ADC values for benign and malignant nodes. ${ }^{36}$

\section{Perfusion Imaging}

An additional means of determining tumor response to treatment is using measures of vascular permeability on DCE images to calculate K trans-values. Similar to DWI, a region of interest is drawn around the enhancing tumor, and this value is used to calculate vascular permeability, or $\mathrm{K}$ trans. Tumors with lower $\mathrm{K}$ trans-values following treatment indicate a favorable response to therapy. ${ }^{37}$ This technique is promising but is not standard in our imaging protocol. More data is required to show its clinical benefit.

\section{Anatomy and Surgical Landmarks}

\section{Anatomy}

Rectum is the last straight segment of the alimentary tract before an angled turn into the anal canal. It extends approximately 12 to $16 \mathrm{~cm}$ from the anocutaneous line (anal verge) that is identified by physical examination as well as with a proctoscope or sigmoidoscope. The sagittal plane offers the best view of the anocutaneous line that is recognized as the inferior margin of the sphincter complex. ${ }^{26}$ Anal verge is an important surgical landmark, and location of inferior border of tumor should be described relative to this line ( - Fig. 6 ).

The anal canal comprises an internal sphincter and external sphincter complex, separated by a thin, fatcontaining intersphincteric plane. The internal sphincter is a continuation of the smooth muscle layer of the rectum. The external sphincter complex begins cranially at the inferior insertion of levator ani muscles and includes the puborectalis muscle and the more inferior external sphincter muscles. ${ }^{23}$ The anatomic relationship of the most inferior aspect of the tumor from the superior border of the anal sphincter and depth of involvement of tumor is of critical importance in MRI evaluation, because it identifies patients who may not be candidates for sphincter preservation surgery. ${ }^{10}$

The rectum is divided into three arbitrary segments of $5 \mathrm{~cm}$ each, as measured from the anal verge and tumors are classified as low $(0-5 \mathrm{~cm})$, mid $(5-10 \mathrm{~cm})$, and high $(10-15 \mathrm{~cm})$ based on distance from the verge. The location where the rectum becomes covered by the peritoneum is considered the rectosigmoid junction and is typically at the S3 level. Internally, the rectal mucosa becomes smooth as it transitions from the rugose mucosa of the sigmoid colon. There are three transverse folds called the superior, middle, and inferior rectal valves of Houston. It is important to know that these folds consist of mucosa and submucosa 


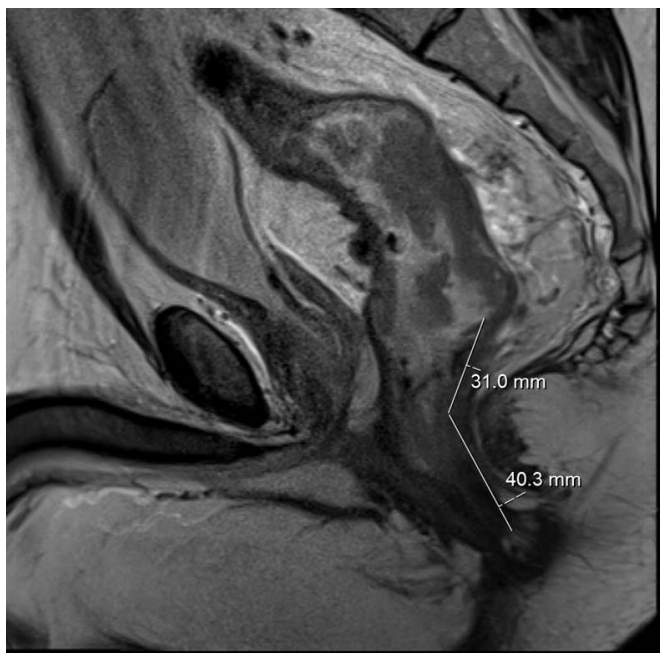

Fig. 6

Measurement of the inferior edge of the tumor from the anal verge.

only. Hence, full-thickness involvement of a fold is a T2 lesion unless the lesion infiltrates the base of the fold. ${ }^{16}$

The rectal wall consists of mucosa, submucosa, and muscularis propria. The thin, outermost layers of connective tissue (subserosa and serosa) that cover most of the colon are lacking in rectum. The outer muscular layer is readily apparent by MRI as a hypointense line. The inner mucosa and submucosa are indistinguishable as a thicker band of slightly higher signal intensity.

\section{Surgical Landmarks}

It is important to understand the anterior peritoneal reflection as it has implications in staging. It separates the intra- and extraperitoneal portions of the rectum and is a well-defined anatomic landmark at laparotomy. ${ }^{38}$ Experienced radiologists identify the anterior peritoneal reflection in more than $80 \%$ of cases. ${ }^{39}$ Normally, the upper third of the rectum is intraperitoneal, the middle third has peritoneum on its anterior aspect, and the lower third is extraperitoneal. In the midsagittal plane, the anterior peritoneal reflection is usually seen as a thin T2 hypointense line on most MRI studies. In men, the tip of the seminal vesicles is a consistent landmark for the location of the most inferior portion of the peritoneal membrane. In women, the location is variable, but the reflection is commonly seen at the uterocervical angle. Midrectal tumors that infiltrate this structure are categorized as T4a disease. The peritoneum attaches in a V-shaped manner onto the anterior aspect of the rectum, an appearance characterized by Brown and colleagues ${ }^{40}$ as the seagull sign. Proper assessment of the anterior peritoneal reflection requires evaluation of both axial and sagittal images ${ }^{41}$ ( Fig. 4).

MRF is an important landmark that surrounds the extraperitoneal portion of rectum and contains the mesorectal fat. It is bordered by Waldeyer's fascia posteriorly and anteriorly by Denonvilliers' fascia in men, which is the equivalent of the rectovaginal septum in women. Denonvilliers' fascia fuses superiorly with the peritoneal reflection. Inferiorly, the MRF fuses with the internal sphincter. MRF is consistently visualized on MRI as a distinct thin T2 hypointense layer surrounding the mesorectum and is best seen on axial images. It is difficult to recognize MRF at the distal and anterior portions of the rectum because of the small amount of fatty tissue.

\section{Magnetic Resonance Imaging Findings}

Anatomic landmarks important to rectal cancer surgery are defined on MRI, which is of use in staging tumors, assessing resectability, planning surgery, and selecting patients for preoperative neoadjuvant therapy. ${ }^{40}$ Modern rectal cancer management is dependent on preoperative staging, and radiologic assessment is a crucial part of this process. Imaging must provide sufficient information to guide preoperative decision making that is reliable and reproducible. ${ }^{42}$

At our institution, we follow a structured synoptic reporting template as recommended by the SAR-DFP on rectal cancer. It is important to have a standardized reporting system to ensure addressing all required characteristics objectively and result in a complete report as is preferred by most treating physicians.

\section{Assessment of Primary Tumor}

Goals of preoperative imaging in rectal cancer include accurate tumor localization, sphincter involvement, depth of mural involvement and extension into mesorectal fat, evaluation of MRF for threatened resection margin, status of peritoneal reflection, identification of extramural vascular invasion (EMVI), and nodal and extranodal metastases. The American Joint Committee on Cancer (tumor-node-metastasis [TNM]) guidelines have been used to develop MRI criteria for the staging of primary rectal tumors ( - Table 2 ). ${ }^{43}$

For purposes of magnetic resonance reporting, the rectum is divided longitudinally into thirds. A tumor is located in the upper third of the rectum if it is more than $10 \mathrm{~cm}$ from the anal verge. Because tumors at this level are generally covered anteriorly by the anterior peritoneal reflection, its relationship (e.g., above, straddles, or below) at this level is important. A tumor arising in the mid third of the rectum is between 5 and $10 \mathrm{~cm}$ from the anal verge, where the rectum is usually encircled by mesorectal fat. A tumor is located in the lower third if it is less than $5 \mathrm{~cm}$ from the anal verge. Tumors residing here may be at or below the sphincter complex, requiring special considerations for reporting on MRI. Craniocaudal location of rectal tumor is frequently significant for surgical planning. Patients with mid and high rectal tumors are usually candidates for sphincter-sparing surgery, whereas those with low rectal tumors have variable candidacy for sphincter preservation.

\section{T Staging}

A schematic representation of $\mathrm{T}$ staging is depicted in $\boldsymbol{- F i g . ~} \mathbf{7}$ and - Table 2 .

\section{T1 Lesions}

These are confined to submucosa and are seen at MRI as hypointense to the surrounding submucosa. Because there is no penetration into the muscular layer, a discrete intact hyperintense ring of submucosa deep to the deep margin of the tumor may be visible. T1 tumors may be difficult to accurately stage with MRI ( - Fig. $\mathbf{8 A}$, B, and C). 


\section{T2 Lesions}

These lesions extend into the hypointense muscularis propria layer, without breaching the outer margin. At MRI, tumor is seen as intermediate signal (higher than muscle and lower than submucosa) that does not extend beyond the outer margin of the muscular layer.

\section{T3 Lesions}

These lesions extend beyond the muscularis layer and into the mesorectal fat but do not invade adjacent structures. Continuity of tumor signal in perirectal fat with the intramural portion of the tumor is crucial. Disruption in the outer muscular layer does not necessitate tumor invasion, because small penetrating wall vessels may give this appearance. ${ }^{44}$ Thin spiculations may also represent peritumoral fibrosis or desmoplastic reaction and are not sensitive or specific in the diagnosis of T3 lesions. ${ }^{45}$ Tumoral nodules within mesorectal fat separate from the tumor itself are also considered as T3 stage. ${ }^{46}$ For T3 tumors, the shortest distance between the most penetrating parts of the tumor and MRF should be measured. The MRF is not circumferential at or above the peritoneal reflection and is best visualized on T2-weighted images as a hypointense line surrounding the mesorectal fat, especially at the proximal and posterior portions of the rectum where fat tissue is more abundant. A tumor-MRF distance of more than $1 \mathrm{~mm}$ is a reliable predictor for negative margins after TME. ${ }^{47}$ In the presence of satellite nodules, the shortest distance between the nodules and MRF should also be reported ${ }^{48}$ ( - Fig. 9A and B).

Table 2 TNM staging of rectal cancer

\begin{tabular}{|c|c|c|}
\hline Primary tumor & Regional lymph nodes & Distant metastasis \\
\hline $\begin{array}{l}\text { TX: primary tumor cannot be } \\
\text { assessed }\end{array}$ & NX: regional lymph nodes cannot be assessed & $\begin{array}{l}\text { M0: no distant metastasis by imaging; no } \\
\text { evidence of tumor in other sites or organs }\end{array}$ \\
\hline T0: no evidence of primary tumor & N0: no regional lymph node metastasis & M1: distant metastasis \\
\hline $\begin{array}{l}\text { Tis: carcinoma in situ, } \\
\text { intramucosal carcinoma }\end{array}$ & N1: metastasis in 1-3 regional lymph nodes & $\begin{array}{l}\text { M1a: metastasis confined to } 1 \text { organ or } \\
\text { site without peritoneal metastasis }\end{array}$ \\
\hline T1: tumor invades submucosa & N1a: metastasis in 1 regional lymph node & $\begin{array}{l}\text { M1b: metastasis to } 2 \text { or more sites or } \\
\text { organs is identified without peritoneal } \\
\text { metastasis }\end{array}$ \\
\hline $\begin{array}{l}\text { T2: tumor invades muscularis } \\
\text { propria }\end{array}$ & N1b: metastasis in 2-3 regional lymph nodes & $\begin{array}{l}\text { M1c: metastasis to the peritoneal surface } \\
\text { is identified alone or with other site or } \\
\text { organ metastases }\end{array}$ \\
\hline $\begin{array}{l}\text { T3: tumor invades through } \\
\text { the muscularis propria into the } \\
\text { pericolorectal tissues }\end{array}$ & $\begin{array}{l}\text { N1c: no regional lymph nodes are positive } \\
\text { but there are tumor deposits in the subserosa, } \\
\text { mesentery or nonperitonealized pericolic or } \\
\text { perirectal / mesorectal tissues }\end{array}$ & \\
\hline $\begin{array}{l}\text { T4a: tumor invades through the } \\
\text { visceral peritoneum }\end{array}$ & N2: metastasis in 4 or more regional lymph nodes & \\
\hline \multirow{2}{*}{$\begin{array}{l}\text { T4b: tumor directly invades or } \\
\text { adheres to other adjacent organs } \\
\text { or structures }\end{array}$} & N2a: metastasis in 4-6 regional lymph nodes & \\
\hline & N2b: metastasis in 7 or more regional lymph nodes & \\
\hline
\end{tabular}

Abbreviation: TNM, tumor-node-metastasis.

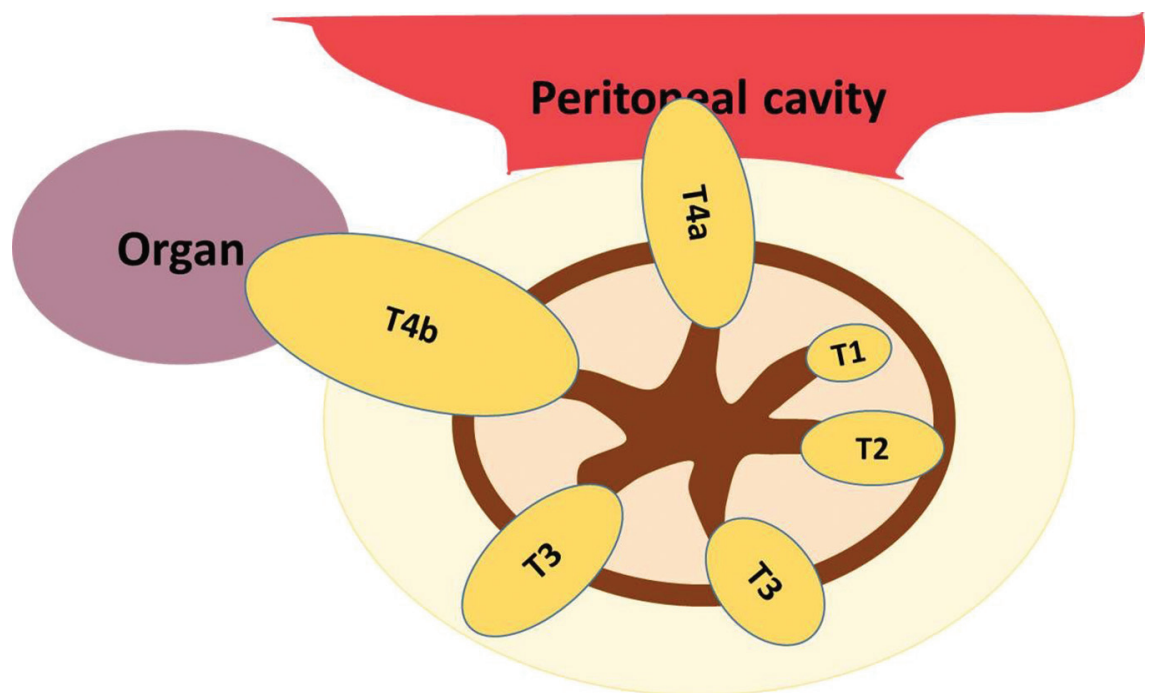

Fig. 7 T-tumor staging of rectal cancer. 

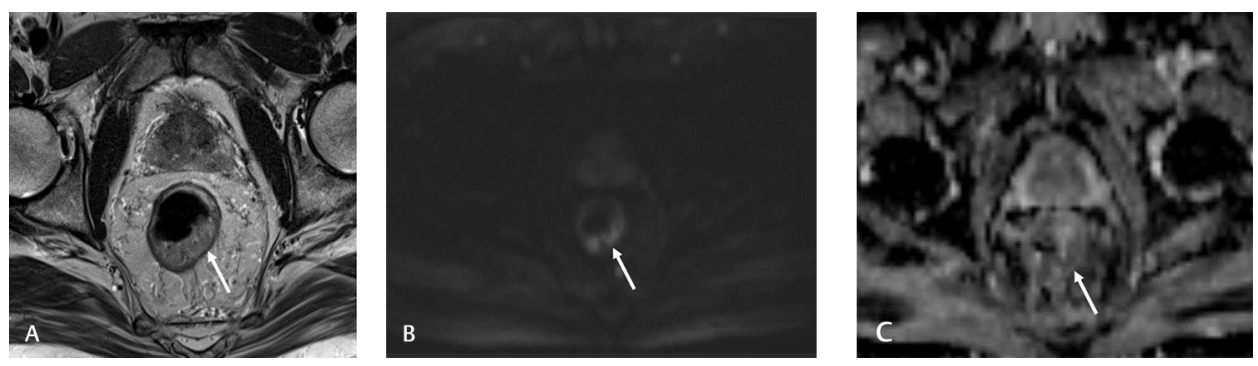

Fig. 8 (A-C) Axial T2, DWI (Diffusion-weighted imaging), and ADC (apparent diffusion coefficient) images. Tumor is of intermediate signal intensity on T2W images and demonstrates restricted diffusion. It does not invade the T2 hypointense muscularis layer (arrow). Postsurgical biopsy confirmed T1 lesion.

\section{T2 versus Early T3 Lesions}

The ability to distinguish $\mathrm{T} 2$ from early $\mathrm{T} 3$ lesions lies in the distinction of speculation of perirectal fat due to peritumoral fibrosis from true tumor infiltration..$^{49}$ MRI does not differentiate well between T2 and early T3 lesions, but this distinction is unlikely to be of clinical significance because patients with early T3 lesions receive little benefit from preoperative neoadjuvant therapy. In tumors where there is speculation of the mesorectal fat, making assessment difficult, reporting tumor as T2/early T3 allows the multidisciplinary team to tailor therapy in the overall assessment of the patient. ${ }^{11,48}$

\section{Extramural Depth of Invasion}

Extramural depth of invasion refers to extension of tumor beyond the muscularis propria and is an independent prognostic factor. The distance of tumor from the CRM is a more robust predictor of local recurrence than T stage. ${ }^{50,51}$ The American Joint Committee on Cancer ${ }^{43}$ suggested an optional stratification of T3 tumors based on the extramural depth of invasion: less than $5 \mathrm{~mm}$, T3a; 5-10 mm, T3b; and more than $10 \mathrm{~mm}$, T3c.

A universal goal of neoadjuvant CCRT is to decrease tumor bulk to provide clear surgical margins at the time of resection, performing surgery with curative intent. In this regard, it is important to identify tumors that involve the MRF or threaten positive margins at the time of surgical excision. Merkel and colleagues ${ }^{50}$ have shown that T3 tumors with greater than $5 \mathrm{~mm}$ of extramural invasion have cancerrelated 5-year survival of only $54 \%$, whereas $\mathrm{T} 3$ tumors with less than $5 \mathrm{~mm}$ of extramural invasion have disease-specific 5 -year survival of $85 \%$ when treatment consists of surgery alone. These findings were reiterated by the Magnetic Resonance Imaging and Rectal Cancer European Equivalence (MERCURY) multi-institutional study, which also affirmed the accuracy of high-resolution MRI in estimating depth of tumor invasion from CRM within $0.5 \mathrm{~mm}$ of measurement of the histopathologic specimen. ${ }^{27}$ Therefore, it is important not only to report an accurate $\mathrm{T}$ stage but also to stratify good and poor prognostic T3 lesions, which can be achieved by determining the depth of extramural involvement beyond muscularis propria. The Radiological society of North America's reporting template includes stratification of T3 tumors into stage T3a through T3c.

\section{T4 Lesions}

If a tumor invades the visceral peritoneum, it is staged as T4a. Therefore, accurate depiction of the peritoneal reflection on MRI is crucial for proper staging of rectal tumors. Invasion into other pelvic structures is classified as stage T4b. Threatened anterior peritoneal reflection margin as well as involvement of other organs usually necessitates preoperative CCRT. Involvement of sidewalls also alters the surgical approach (-Figs. 10, 11A and B).

\section{Assessment of Mesorectal Fascia}

The relationship of the tumor to MRF is crucial for surgical planning and can reliably be assessed with MRI. The shortest distance should be regarded as the closest distance from either tumor margin, tumoral deposit or lymph node, or tumor thrombus. Because of the lack of sensitivity and specificity of peritumoral speculation in the mesorectal fat, it
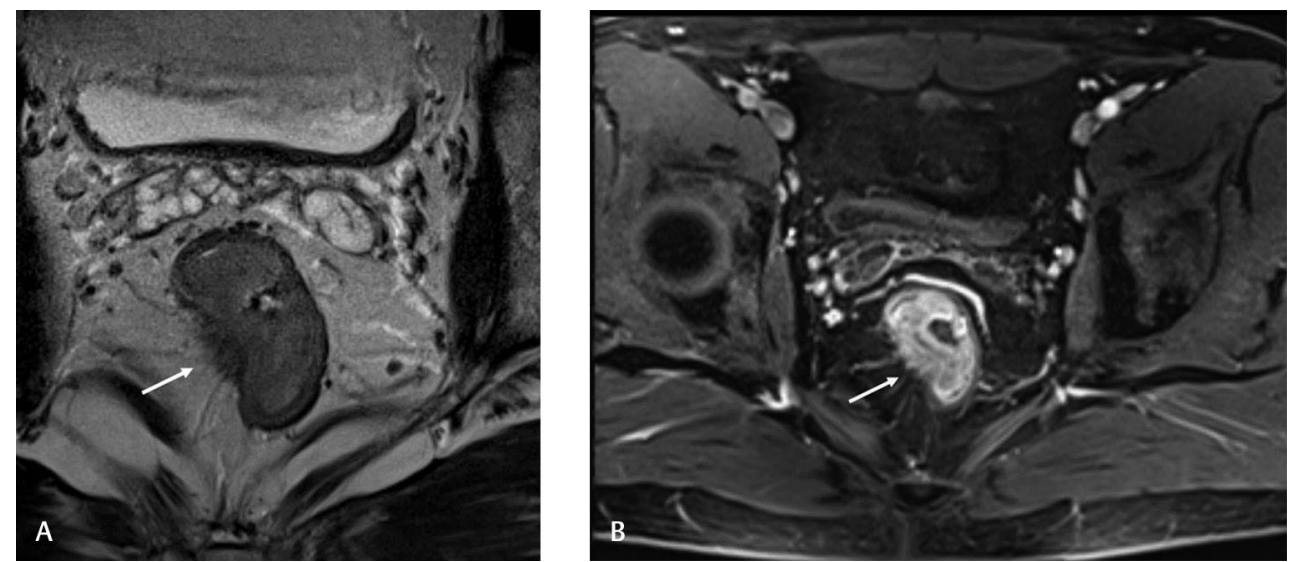

Fig. 9 (A, B) Axial T2 and postcontrast T1 images show spiculations along the tumor margin extending into the perirectal fat (arrows). This was thought to represent desmoplastic reaction associated with the tumor and staged as a T2 lesion on MRI. Postsurgical biopsy showed T3 disease. 
is advisable to report the distance from the speculation to CRM and most penetrating part of tumor to CRM separately. ${ }^{10}$ There is some controversy regarding distance of tumor from the MRF that predicts a safe surgical margin at the MRF. Beets-Tan and colleagues ${ }^{49}$ have determined that histologically a tumor-free margin of $1 \mathrm{~mm}$ is predictable when the distance from tumor to MRF is $5 \mathrm{~mm}$ or greater on imaging. This is in contradistinction to an early study by Brown and colleagues, ${ }^{52}$ wherein the distance from tumor to MRF as measured on MRI was determined to be equivalent to histopathology. Similar findings were later emphasized by the MERCURY study group. ${ }^{25}$ Notwithstanding, both groups have demonstrated MRI to be a highly accurate tool in the assessment of extramural depth of invasion and involvement of MRF on final histopathology.

A more recent study by Taylor and Colleagues ${ }^{47}$ compared measurements of 1,2 , and $5 \mathrm{~mm}$, and assessed whether more patients with subsequent local recurrence were identified by increasing the distance from 1 to 2 or $5 \mathrm{~mm}$. This study showed no significant difference in positive resection margins or recurrence rate by increasing the cutoff. Thus, use of margins larger than $1 \mathrm{~mm}$ may lead to patients receiving unnecessary neoadjuvant CRT. Indeed, this study follows up with patients from the MERCURY trial and notes that had the trial used $5 \mathrm{~mm}$ instead of $1 \mathrm{~mm}$ as its cutoff, 89 of the 216 patients would have been given CRT to prevent seven local recurrences. At our institution, we consider a measured distance of $1 \mathrm{~mm}$ or less on high-resolution T2-weighted images to be indicative of CRM involvement.

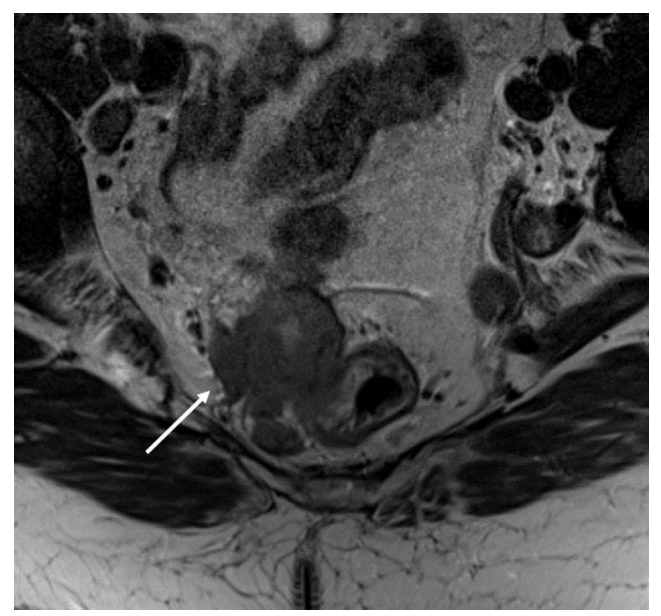

Fig. 10 Axial T2 image showing T4 disease-tumor extending beyond the margin of the mesorectal fascia (arrow).
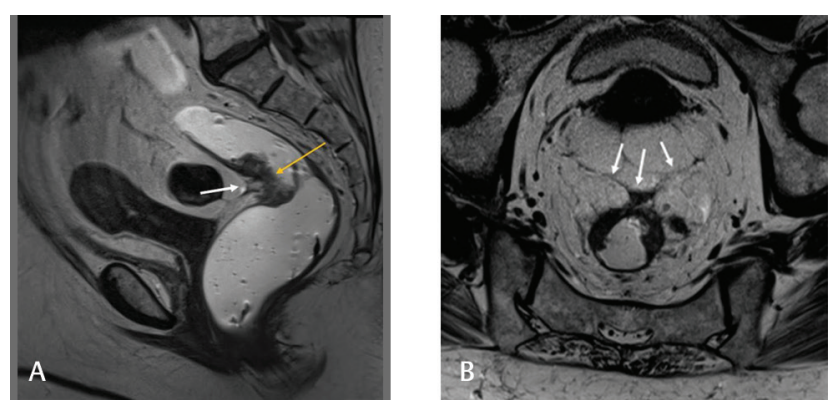

Fig. 11 (A, B) Sagittal and coronal T2W images showing tumor (arrow) straddling the anterior peritoneal reflection (arrows).

\section{Extramural Vascular Invasion}

EMVI refers to invasion of large vessels deep to the muscular layer. EMVI is not included in staging but has prognostic significance. Magnetic resonance assessment of EMVI is moderately sensitive (62\%) and highly specific (88\%).53 EMVI on MRI should be called present when there is obvious irregularity of a vessel or intermediate intraluminal signal in vessels close to tumor replacing flow voids. EMVI is absent when there is no tumor extension in close proximity to vascular structure; pattern of tumor invasion through muscularis propria is not nodular. If there is stranding near a vessel, EMVI is considered absent if the vessels are normal in caliber and signal intensity. Studies on EMVI have concluded that even in patients with a good response to chemoradiotherapy followed by curative surgery, EMVI may predict poor disease-free survival outcomes ${ }^{54}$ ( Fig. 12A, B, and C).

\section{Low Rectal Tumors}

Low rectal cancers are generally defined as lowest margin of tumor within $5 \mathrm{~cm}$ of the anal verge, increasing the likelihood of involvement of the anal sphincter complex. Relationship of tumor to sphincter complex and the ability to achieve clear distal margins not only determines the surgical approach but is the key to success of surgery, because it is critical to achieving a good functional and oncologic outcome (-Fig. 13).

Examination of the sagittal and coronal images is essential to avoid overstaging from apparent levator involvement on the axial images. ${ }^{5}$ The depth of invasion for tumors at or below puborectalis should be described. When evaluating a low-lying tumor for resection, the distal resection margin is the most important consideration for deciding which patients can receive sphincter sparing surgery versus $A P R .{ }^{55}$ In the past, a distal resection margin of $5 \mathrm{~cm}$ was recommended, and low-lying tumors often required sphincter removal. Recent literature suggests that a margin of $2 \mathrm{~cm}$ in a nonirradiated tumor or $1 \mathrm{~cm}$ following treatment has a recurrence rate similar to the more generous 5 - $\mathrm{cm}$ margin previously used. ${ }^{55}$ Some studies have even suggested that a margin of $5 \mathrm{~mm}$ in carefully selected patients could provide acceptable results. ${ }^{56}$ Sphincter sparing surgery can be an important option, especially in younger patients with lower-stage tumors.

\section{Mucinous Tumors}

Mucinous rectal carcinoma is a pathologic subtype of rectal adenocarcinoma characterized by production of extracellular mucin. These tumors tend to have a higher pathologic stage at the time of diagnosis, a greater tendency for metastasis, and local recurrence and unfavorable prognosis. Identification of mucinous tumors is possible due to their intrinsic increased T2 signal relative to nonmucinous cancer. This may facilitate surgical management, because mucinous tumors are highly infiltrative ( - Fig. 14A and B).

\section{Nodal Staging}

Mesorectal lymph nodes constitute the initial nodal drainage of rectal tumors, followed by superior rectal and inferior mesenteric nodes. ${ }^{57}$ Extramesorectal nodes are most commonly found along the middle rectal artery, internal 

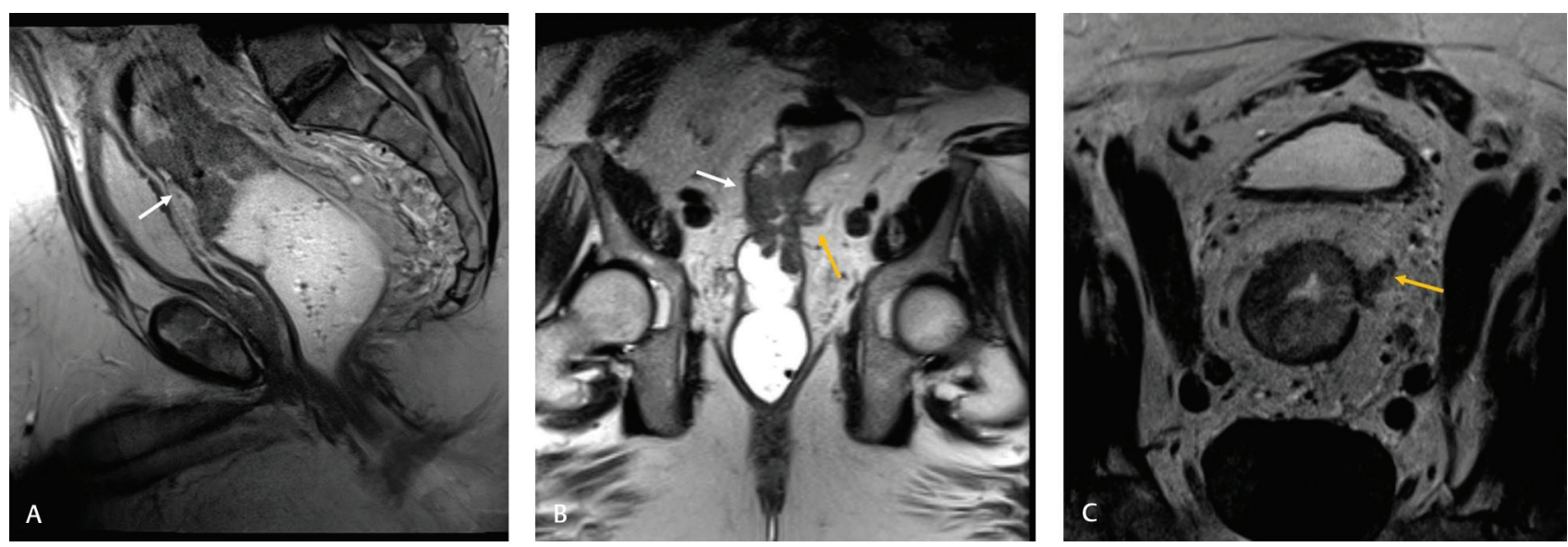

Fig. 12 (A-C) Sagittal, coronal, and axial T2 images showing T3 disease with extramural vascular invasion (EMVI) in the form of nodular infiltration of vessels at the left margin (arrows).

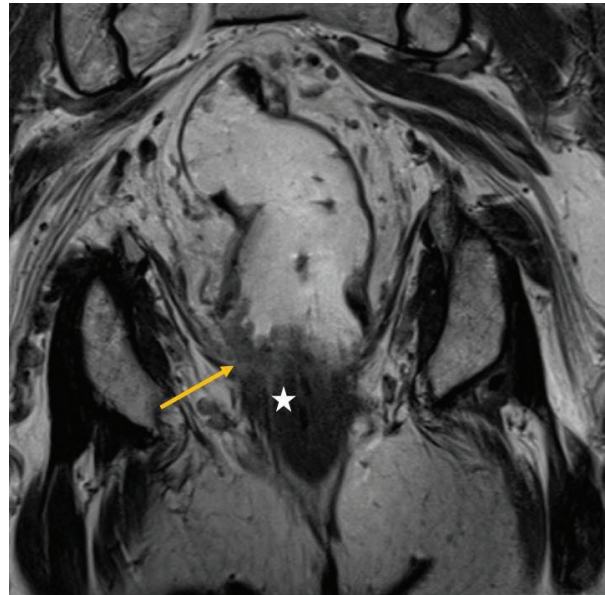

Fig. 13 Coronal T2 image showing low rectal tumor (arrow) reaching the upper margin of internal sphincter (star).
By TNM staging, N1 is defined by one to three positive regional lymph nodes, and $\mathrm{N} 2$ is defined by four or more positive nodes. Assessment of mesorectal nodes is important for surgical planning so that surgeons can stay clear of these nodes during TME, although they should be completely excised. Additionally, the presence of involved lateral nodes at the pelvic sidewall is significant in terms of prognosis and treatment planning. In the United States, nodes outside the MRF along the pelvic sidewall are not routinely resected. However, if involvement of these nodes can be established preoperatively, it is important to modify the treatment approach to avoid local recurrence. ${ }^{23,60}$ Involved extramesorectal lymph nodes can be targeted with a widened field for preoperative radiation therapy and extended surgical resection, so individual extramesorectal nodes merit comment at initial staging MRI. Moreover, it is important to
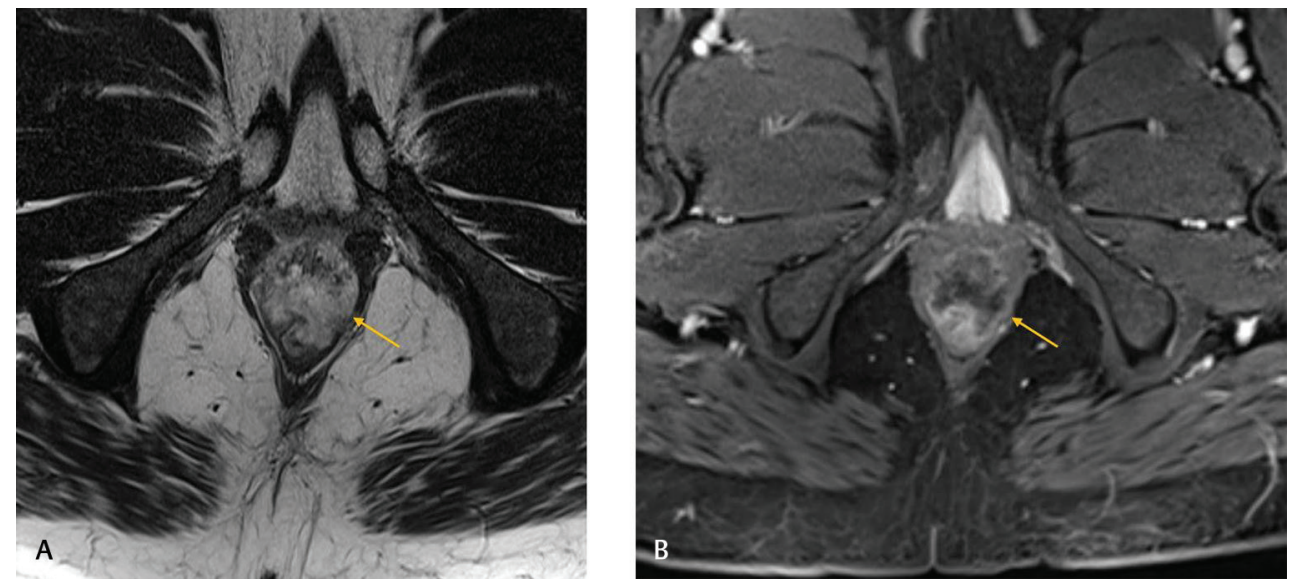

Fig. 14 (A, B) Axial T2 images showing a low rectal tumor that is hyperintense on T2W images and poorly enhancing on postcontrast images (arrows).

iliac chain, obturator, median sacral, and, less commonly, external or common iliac nodes. Inguinal nodes represent a nonregional site of nodal metastasis and are more commonly identified in low rectal cancers. Inguinal nodes are associated with a poor prognosis and have a high association with diffuse disease. ${ }^{58,59}$ evaluate these nodes for the purposes of staging, because malignant external iliac and superficial inguinal nodes imply stage M1 disease.

It is well established that nodal size is of limited value in assessing for the presence of metastasis. The most frequently used size criterion for distinguishing malignant from 
nonmalignant nodes $(5 \mathrm{~mm})$ has a sensitivity of $68 \%$ and a specificity of approximately $78 \% .{ }^{15,61}$ The limited accuracy of nodal size is likely related to the fact that 30 to $50 \%$ of metastases in rectal cancer occur in nodes that are less than $5 \mathrm{~mm}$. In several studies, however, lymph nodes greater than $8 \mathrm{~mm}$ were highly specific for metastatic involvement, and this has been accepted as a predictor of nodal status based on size alone. ${ }^{61-63}$ This cutoff value for size compromises sensitivity and specificity.

Nodal margins and internal nodal characteristics are the most reliable indicators of malignancy. ${ }^{61}$ Features suggestive of malignancy include irregular or spiculated nodal margins and heterogeneous signal intensity. The evaluation of these features requires high-resolution images that cover all nodes of importance, including superior rectal and pelvic sidewall adenopathy. Craniocaudal localization and clock-face localization of suspicious lymph nodes relative to the tumor are necessary; for mesorectal nodes, the distance to the MRF should also be measured.

DWI is a useful adjunct to conventional T2 imaging, increasing the sensitivity of small lymph nodes. A study by Mir and colleagues ${ }^{64}$ has shown that DWI detects a significant number of sub-centimeter lymph nodes not identified by T2 imaging, thus improving detection of small nodal metastases and negative predictive value. Differentiation of benign and malignant sub-centimeter nodes, however, is not possible with DWI.

Use of nanoparticle contrast media, such as ultra small super-paramagnetic iron oxide (USPIO) particles, has been studied for nodal assessment. The magnetic properties of USPIO particles generate changes in $\mathrm{T} 2$ and $\mathrm{T} 2{ }^{*}$ signal intensity. In healthy lymph nodes, the particles are phagocytosed, leading to decreased $\mathrm{T} 2$ signal. Tumor cells prevent the uptake of nanoparticles, resulting in increased signal in involved nodes. ${ }^{65}$ Use of USPIO particles is limited by availability in some countries and by timing considerations in others, as it must be administered 24 to 36 hours before performing the magnetic resonance examination. Currently, the only U.S. Food and Drug Administration approved and commercially available USPIO is ferumoxytol. ${ }^{66}$

Gadofosveset is a gadolinium-based intravascular contrast agent that reversibly binds to albumin providing extended intravascular enhancement compared with existing extracellular magnetic resonance contrast agents and was originally marketed as a magnetic resonance angiography agent. Studies by Lambregts and colleagues ${ }^{67,68}$ have determined that imaging with Gadofosveset provides a high performance in diagnosis of malignant nodes. Benign nodes take up contrast, whereas malignant nodes do not, with promising results. Although larger validation studies have not been completed, use of gadofosveset as a node-specific contrast agent may further increase the specificity of nodal staging by MRI. However, the interpretation of these findings for nodes in the superior mesorectum or those in the vicinity of vessels is challenging, and the presence of micrometastases cannot be ruled out. ${ }^{69}$

FDG-PET has high specificity (85-95\%) for the identification of malignant nodes, but its role in nodal staging is limited because of low sensitivity (29-63\%) and the inability to accurately localize the involved nodes. ${ }^{70,71}$ It is more suitable for the assessment of extramesorectal nodes because high uptake of tracer by the primary tumor may prevent the visualization of mesorectal nodes. ${ }^{72}$ Application of CT with PET improves the ability of the modality for anatomic localization. PET-CT has shown 63 to $70 \%$ accuracy for the detection of regional lymph node metastasis, and the combination of highresolution MRI with PET-CT increases the accuracy up to $90 \%{ }^{73}$

\section{Assessment of Treatment Response}

Preoperative neoadjuvant CRT has improved the survival of patients possibly by increasing the CRM-negative resections. ${ }^{74}$ In addition, it may enable sphincter preserving resection in patients with low rectal tumors through downstaging of locally advanced disease. Tumor restaging involves correlation of posttreatment images with the pretreatment images with respect to all elements assessed in the initial staging and necessitates image acquisition with almost the same protocol and in the same planes ${ }^{75}$ ( - Fig. 15A, B, C, D, and E).

Tumor characteristics should be reported in detail, including morphologic appearance of the tumor, length of tumor, and distance from anal verge. Depth of maximum extramural spread and fibrosis should be discussed. It is also of particular importance to comment on involvement of suspicious extramesorectal lymph nodes and possible anterior peritoneal reflection involvement at the time of restaging. One of the most important parameters in restaging is reassessment of the MRF. The persistence of EMVI after CRT can be detected on MRI and, regardless of the final pathologic staging, may predict a higher risk of metastatic disease and an overall shorter disease-free survival. ${ }^{76}$ Therefore, EMVI status after CRT may possibly be used as an imaging biomarker for counseling patients for postoperative chemotherapy or more intensive surveillance ( - Fig. 16A and B).

The accuracy of MRI for restaging, however, is generally lower than that for initial staging mainly owing to overstaging of nodal disease, failure to differentiate tumoral infiltration or residual tumor from desmoplastic reaction or radiation fibrosis, and misinterpretation of radiation proctitis as local invasion. ${ }^{77}$ Also, MRI is not reliable for confirming complete response because of its inability to detect microscopic residual tumor or mucin lakes that can be detected at histopathology; therefore, caution should be exercised in claiming "complete response" on post-CRT MRI reports. Evaluation of mucinous adenocarcinomas on posttreatment MRI is also considerably challenging because these tumors remain hyperintense on $\mathrm{T} 2$-weighted imaging regardless of their response to treatment. ${ }^{78}$

DCE-MRI has been evaluated in the restaging of rectal cancer with variable results. Pretreatment perfusion index values and slope of contrast medium enhancement curve help identify responders. ${ }^{79}$ In a recent systematic review by Dijkhoff and colleagues, the authors concluded that DCE-MRI is promising mainly for prediction and assessment of response to CRT, where a high pre-CRT $\mathrm{K}$ trans with a decrease in $\mathrm{K}$ trans are significant predictors of response. ${ }^{34} \mathrm{~K}$ trans is the most thoroughly investigated parameter and correlates with angiogenetic activity in tumor and tumor aggressiveness. This 

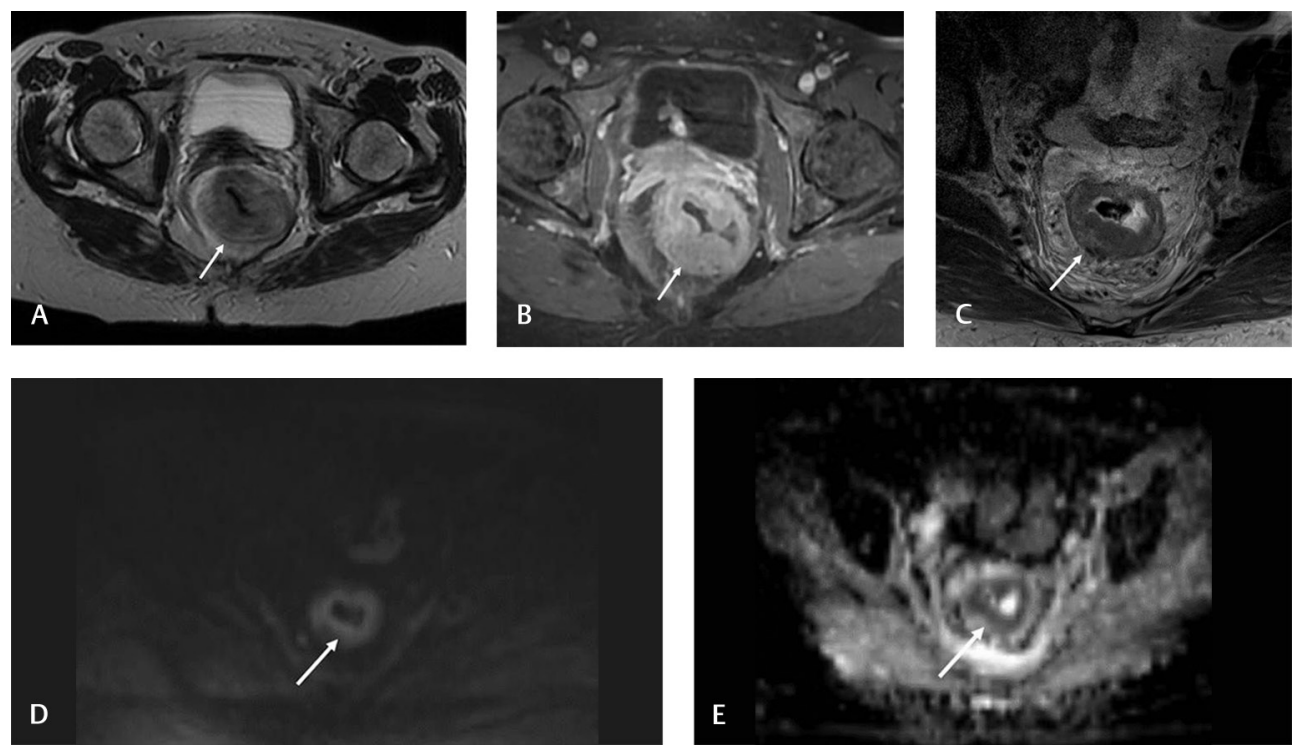

Fig. 15 (A, B) Pretreatment tumor (arrow) on T2-weighted image and diffusion-weighted imaging (DWI). Posttreatment images. (C-E) Significant residual disease suggesting partial treatment response.
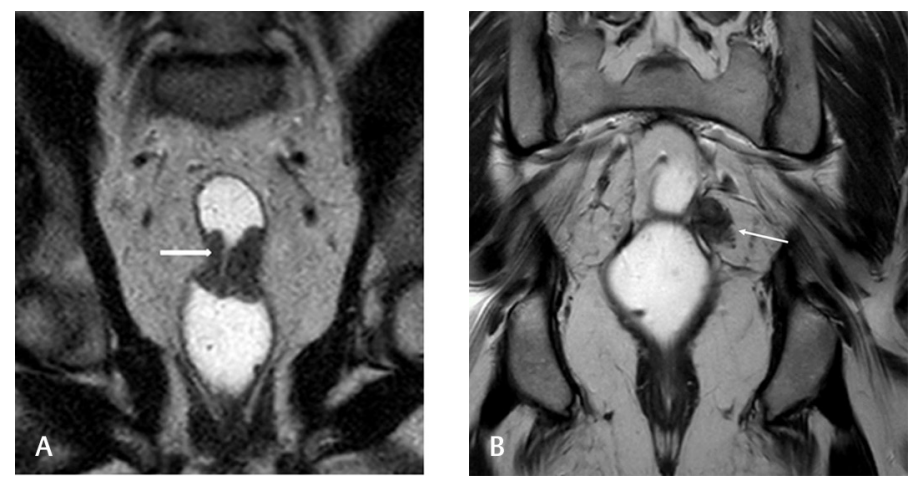

Fig. 16 (A, B) Patient with prior history of LAR. Follow-up MRI shows soft tissue nodule at the anastomotic site suggestive of recurrence.

systematic review concludes that despite the large variability in DCE studies in rectal cancer, there seems a value for DCEMRI in the staging and restaging of rectal cancer.

DWI provides functional information that can be correlated with changes at the cellular level in response to treatment. After CRT, the decrease in cellularity and development of fibrosis or necrosis in responders result in an increase in diffusion and increase in the ADC value. ${ }^{80} \mathrm{~A}$ meta-analysis study has shown that DWI is more sensitive than (62-94\%) and is almost as specific as (74-91\%) conventional MRI in restaging rectal tumors after CRT. ${ }^{81}$ This has been further corroborated more recently by Enkhbaatar and colleagues ${ }^{82}$ who studied response to neoadjuvant therapy in patients with locally advanced rectal cancer by using MR-ADC histogram analysis. The authors concluded that in patients with advanced rectal cancer, post-CRT skewness of $\mathrm{ADC}$ histogram and percentage change in $\mathrm{ADC}$ were useful for predicting a favorable response to neoadjuvant CRT. Use of quantitative $\mathrm{ADC}$ is not advisable as routine, because current software algorithms for ADC value calculation preclude reproducible measurements across vendor platforms. ${ }^{10}$

DWI has also been used to predict response to treatment and tumor aggressiveness. At initial staging MRI, a high ADC value corresponds to rapid diffusion in the necrotic area of a cancer and indicates a more aggressive tumor. This predicts a poor response to CRT. ${ }^{83,84}$

Studies have evaluated the measurement of tumoral volume on both T2 and DWI for assessment of treatment response. As per Quaia and colleagues, ${ }^{85}$ the percentage change in tumoral volume at T2-weighted MRI and DWI can differentiate responders from nonresponders in patients with locally advanced rectal cancers after neoadjuvant CRT. Similar findings are reported by Birlik et al who conclude that percentage tumor volume regression after preoperative CRT was correlated with good response and aided differentiating responders and nonresponders. ${ }^{86}$

More recently, T2-weighted-based radiomics analysis has been studied to assess treatment response after neoadjuvant therapy, when compared with DWI and qualitative T2 imaging for diagnosis of complete response. ${ }^{87}$ Horvat and colleagues concluded that $\mathrm{T} 2$-weighted-based radiomics showed better classification performance compared with qualitative assessment at T2-weighted and DWI for diagnosing pathologic complete response in patients with locally advanced rectal cancer after CRT. 
One aspect that has been inconsistent in assessment of treatment response is the timeline of MRI following CRT. While some studies have subjected patients to MRI only after completion of a few cycles of treatment, a few studies have had the patients undergo MRI once or twice during early phase of radiation. ${ }^{83,88}$ Recently, a few studies have performed sequential MRIs to assess treatment effect starting as early as first week of therapy. In a recently published study from 2017, Lambregts et $\mathrm{al}^{89}$ performed sequential MRIs before, during, and after preoperative chemoradiotherapy in a small cohort of 13 locally advanced rectal cancer patients. The authors found that rectal tumor volumes decreased significantly after each week of radiation. The main rectal tumor regression in their study occurred during the first half of CRT with speed of shrinkage decreasing over the course, with a peak response between weeks 2 and 3 . These results could be appreciated both quantitatively (in tumor volumes) and qualitatively while visually assessing the tumor. Lambrecht et al ${ }^{88}$ measured tumor volume after 2 to 3 weeks and found that the percentage decrease in volume compared with baseline was significantly correlated with T-downstaging.

These results indicate that early signs of response can be captured with routine morphologic imaging both by means of qualitative (visual) interpretation and by quantitative tumor volume measurements. These early signs of response may provide an indication of the final response outcome, particularly when assessed mid-CRT, when early tumor regression appears to be most pronounced. This timeline can be of great clinical importance when aiming to identify patients for more personalized neoadjuvant strategies, because it can help in deciding the best time point to evaluate early treatment response. For further validation of this timeline, it would be interesting to combine these findings with early changes in functional magnetic resonance tumor parameters such as those derived from diffusion-weighted or DCE magnetic resonance sequences.

Tumor regression grading (TRG) systems that aim to categorize the amount of regressive changes after cytotoxic treatment are used by histologists and refer to the amount of therapy-induced fibrosis in relation to residual tumor or the estimated percentage of residual tumor in relation to the previous tumor site. Modified Dowark classification is one of the widely used histologic grading systems, and many authors have now proposed analogous MRI-TRG systems to match the histopathology as well..$^{90,91}$

\section{Summary}

As an important member of multidisciplinary team to approach rectal cancer treatment, radiologists play a crucial role. Providing accurate treatment requires a pretherapy prediction of risk for local recurrence, which is accomplished with staging MRI. MRI also provides additional prognostic information by assessment of extramural depth of invasion, EMVI, and CRM, establishing its essential role in the preoperative assessment of rectal cancer. In addition, MRI through use of specialized sequences such as DWI helps in assessing treatment response, especially as conservative management techniques (wait-andsee approach) become more clinically relevant.

\section{Conflict of Interest}

None declared.

\section{References}

1 Siegel R, Naishadham D, Jemal A. Cancer statistics, 2013. CA Cancer J Clin 2013;63(1):11-30

2 American Cancer Society. Colorectal Cancer Facts and Figures 2011-2013. Atlanta, GA: American Cancer Society; 2011

3 Sauer R, Becker H, Hohenberger W, et al; German Rectal Cancer Study Group. Preoperative versus postoperative chemoradiotherapy for rectal cancer. N Engl J Med 2004;351 (17):1731-1740

4 Heald RJ, Moran BJ, Ryall RD, Sexton R, MacFarlane JK. Rectal cancer: the Basingstoke experience of total mesorectal excision, 1978-1997. Arch Surg 1998;133(8):894-899

5 Shihab OC, Moran BJ, Heald RJ, Quirke P, Brown G. MRI staging of low rectal cancer. Eur Radiol 2009;19(3):643-650

6 Kosinski L, Habr-Gama A, Ludwig K, Perez R. Shifting concepts in rectal cancer management: a review of contemporary primary rectal cancer treatment strategies. CA Cancer J Clin 2012;62(3):173-202

7 Hermanek P, Junginger T. The circumferential resection margin in rectal carcinoma surgery. Tech Coloproctol 2005;9(3):193199, discussion 199-200

8 Quirke P, Durdey P, Dixon MF, Williams NS. Local recurrence of rectal adenocarcinoma due to inadequate surgical resection. Histopathological study of lateral tumour spread and surgical excision. Lancet 1986;2(8514):996-999

9 Liu Q, Luo D, Cai S, Li Q, Li X. Circumferential resection margin as a prognostic factor after rectal cancer surgery: a large population-based retrospective study. Cancer Med 2018;7(8):3673-3681 [Epub ahead of print]

10 Furey E, Jhaveri KS. Magnetic resonance imaging in rectal cancer. Magn Reson Imaging Clin N Am 2014;22(2):165-190, v-vi

11 User's Guide for the synoptic MRI report for Pre-operative staging of Rectal Cancer 2015, 1-25. https://archive.cancercare.on.ca. A joint initiative of Cancer Care Ontario and the Canadian Cancer Society

12 Garcia-Aguilar J, Pollack J, Lee SH, et al. Accuracy of endorectal ultrasonography in preoperative staging of rectal tumors. Dis Colon Rectum 2002;45(1):10-15

13 Halefoglu AM, Yildirim S, Avlanmis O, Sakiz D, Baykan A. Endorectal ultrasonography versus phased-array magnetic resonance imaging for preoperative staging of rectal cancer. World J Gastroenterol 2008;14(22):3504-3510

14 Puli SR, Bechtold ML, Reddy JBK, Choudhary A, Antillon MR, Brugge WR. How good is endoscopic ultrasound in differentiating various $\mathrm{T}$ stages of rectal cancer? Meta-analysis and systematic review. Ann Surg Oncol 2009;16(2):254-265

15 Bipat S, Glas AS, Slors FJM, Zwinderman AH, Bossuyt PM, Stoker J. Rectal cancer: local staging and assessment of lymph node involvement with endoluminal US, CT, and MR imaging-a meta-analysis. Radiology 2004;232(3):773-783

16 Beaumont C, Pandey T, Gaines Fricke R, Laryea J, Jambhekar K. MR evaluation of rectal cancer: current concepts. Curr Probl Diagn Radiol 2013;42(3):99-112

17 Beets-Tan RG, Beets GL. Rectal cancer: review with emphasis on MR imaging. Radiology 2004;232(2):335-346

18 Maizlin ZV, Brown JA, So G, et al. Can CT replace MRI in preoperative assessment of the circumferential resection margin in rectal cancer? Dis Colon Rectum 2010;53(3):308-314

19 Eglinton T, Luck A, Bartholomeusz D, Varghese R, Lawrence M. Positron-emission tomography/computed tomography (PET/ $\mathrm{CT}$ ) in the initial staging of primary rectal cancer. Colorectal Dis 2010;12(7):667-673 
20 Balyasnikova S, Brown G. Optimal imaging strategies for rectal cancer staging and ongoing management. Curr Treat Options Oncol 2016;17(6):32

21 Gollub MJ, Arya S, Beets-Tan RG, et al. Use of magnetic resonance imaging in rectal cancer patients: Society of Abdominal Radiology (SAR) rectal cancer disease-focused panel (DFP) recommendations 2017. Abdom Radiol (NY) 2018;43(11):2893-2902

22 Slater A, Halligan S, Taylor SA, Marshall M. Distance between the rectal wall and mesorectal fascia measured by MRI: Effect of rectal distension and implications for preoperative prediction of a tumour-free circumferential resection margin. Clin Radiol 2006;61(1):65-70

23 Kaur H, Choi H, You YN, et al. MR imaging for preoperative evaluation of primary rectal cancer: practical considerations. Radiographics 2012;32(2):389-409

24 Dewhurst C, Rosen MP, Blake MA, et al. ACR Appropriateness Criteria pretreatment staging of colorectal cancer. J Am Coll Radiol 2012;9(11):775-781

25 Brown G, Daniels IR. Preoperative staging of rectal cancer: the MERCURY research project. Recent Results Cancer Res 2005;165:58-74

26 Torkzad MR, Påhlman L, Glimelius B. Magnetic resonance imaging (MRI) in rectal cancer: a comprehensive review. Insights Imaging 2010;1(4):245-267

27 MERCURY Study Group. Extramural depth of tumor invasion at thin-section MR in patients with rectal cancer: results of the MERCURY study. Radiology 2007;243(1):132-139

28 Suzuki C, Torkzad MR, Tanaka S, et al. The importance of rectal cancer MRI protocols on interpretation accuracy. World J Surg Oncol 2008;6:89

$29 \mathrm{Kim} \mathrm{H}$, Lim JS, Choi JY, et al. Rectal cancer: comparison of accuracy of local-regional staging with two- and three-dimensional preoperative 3-T MR imaging. Radiology 2010;254(2):485-492

30 Jao SY, Yang BY, Weng H, Yeh CH, Lee LW. Evaluation of gadolinium-enhanced T1-weighted magnetic resonance imaging in the preoperative assessment of local staging in rectal cancer. Colorectal Dis 2010;12(11):1139-1148

31 Gollub MJ, Lakhman Y, McGinty K, et al. Does gadolinium-based contrast material improve diagnostic accuracy of local invasion in rectal cancer MRI? A multireader study. AJR Am J Roentgenol 2015;204(2):W160-7

32 Vliegen RF, Beets GL, von Meyenfeldt MF, et al. Rectal cancer: MR imaging in local staging-is gadolinium-based contrast material helpful? Radiology 2005;234(1):179-188

33 Beets-Tan RGH, Lambregts DMJ, Maas M, et al. Magnetic resonance imaging for clinical management of rectal cancer: Updated recommendations from the 2016 European Society of Gastrointestinal and Abdominal Radiology (ESGAR) consensus meeting. Eur Radiol 2018;28(4):1465-1475

34 Dijkhoff RAP, Beets-Tan RGH, Lambregts DMJ, Beets GL, Maas $M$. Value of DCE-MRI for staging and response evaluation in rectal cancer: a systematic review. Eur J Radiol 2017;95:155-168

35 Lim KS, Tan CH. Diffusion-weighted MRI of adult male pelvic cancers. Clin Radiol 2012;67(9):899-908

36 Mizukami Y, Ueda S, Mizumoto A, et al. Diffusion-weighted magnetic resonance imaging for detecting lymph node metastasis of rectal cancer. World J Surg 2011;35(4):895-899

37 Rudisch A, Kremser C, Judmaier W, Zunterer H, DeVries AF. Dynamic contrast-enhanced magnetic resonance imaging: a non-invasive method to evaluate significant differences between malignant and normal tissue. Eur J Radiol 2005;53(3):514-519

38 Dujovny N, Quiros RM, Saclarides TJ. Anorectal anatomy and embryology. Surg Oncol Clin N Am 2004;13(2):277-293

39 Gollub MJ, Maas M, Weiser M, et al. Recognition of the anterior peritoneal reflection at rectal MRI. AJR Am J Roentgenol 2013;200(1):97-101
40 Brown G, Kirkham A, Williams GT, et al. High-resolution MRI of the anatomy important in total mesorectal excision of the rectum. AJR Am J Roentgenol 2004;182(2):431-439

41 Salerno G, Daniels IR, Moran BJ, Wotherspoon A, Brown G. Clarifying margins in the multidisciplinary management of rectal cancer: the MERCURY experience. Clin Radiol 2006;61(11):916-923

42 Bhoday J, Balyasnikova S, Wale A, Brown G. How should imaging direct/orient management of rectal cancer? Clin Colon Rectal Surg 2017;30(5):297-312

43 American Joint Committee on Cancer. AJCC Cancer Staging Manual. 7th ed. New York, NY: Springer; 2010

44 Taylor FG, Swift RI, Blomqvist L, Brown G. A systematic approach to the interpretation of preoperative staging MRI for rectal cancer. AJR Am J Roentgenol 2008;191(6):1827-1835

45 Brown G, Radcliffe AG, Newcombe RG, Dallimore NS, Bourne MW, Williams GT. Preoperative assessment of prognostic factors in rectal cancer using high-resolution magnetic resonance imaging. Br J Surg 2003;90(3):355-364

46 Compton CC, Greene FL. The staging of colorectal cancer: 2004 and beyond. CA Cancer J Clin 2004;54(6):295-308

47 Taylor FG, Quirke P, Heald RJ, et al; MERCURY study group. One millimetre is the safe cut-off for magnetic resonance imaging prediction of surgical margin status in rectal cancer. $\mathrm{Br} \mathrm{J}$ Surg 2011;98(6):872-879

48 Jhaveri KS, Hosseini-Nik H. MRI of rectal cancer: an overview and update on recent advances. AJR Am J Roentgenol 2015;205(1):W42-55

49 Beets-Tan RG, Beets GL, Vliegen RF, et al. Accuracy of magnetic resonance imaging in prediction of tumour-free resection margin in rectal cancer surgery. Lancet 2001;357(9255) :497-504

50 Merkel S, Mansmann U, Siassi M, Papadopoulos T, Hohenberger W, Hermanek P. The prognostic inhomogeneity in pT3 rectal carcinomas. Int J Colorectal Dis 2001;16(5):298-304

51 Pollheimer MJ, Kornprat P, Pollheimer VS, et al. Clinical significance of pT sub-classification in surgical pathology of colorectal cancer. Int J Colorectal Dis 2010;25(2):187-196

52 Brown G, Richards CJ, Newcombe RG, et al. Rectal carcinoma: thin-section MR imaging for staging in 28 patients. Radiology 1999;211(1):215-222

53 Smith NJ, Barbachano Y, Norman AR, Swift RI, Abulafi AM, Brown G. Prognostic significance of magnetic resonance imaging-detected extramural vascular invasion in rectal cancer. Br J Surg 2008;95(2):229-236

54 Cho MS, Park YY, Yoon J, et al. MRI-based EMVI positivity predicts systemic recurrence in rectal cancer patients with a good tumor response to chemoradiotherapy followed by surgery. J Surg Oncol 2018;117(8):1823-1832

55 Park IJ, Kim JC. Adequate length of the distal resection margin in rectal cancer: from the oncological point of view. J Gastrointest Surg 2010;14(8):1331-1337

56 Bujko K, Rutkowski A, Chang GJ, Michalski W, Chmielik E, Kusnierz $\mathrm{J}$. Is the $1-\mathrm{cm}$ rule of distal bowel resection margin in rectal cancer based on clinical evidence? A systematic review. Ann Surg Oncol 2012;19(3):801-808

57 Engelen SM, Beets-Tan RG, Lahaye MJ, Kessels AG, Beets GL. Location of involved mesorectal and extramesorectal lymph nodes in patients with primary rectal cancer: preoperative assessment with MR imaging. Eur J Surg Oncol 2008;34(7):776-781

58 Wang C, Zhou ZG, Yu YY, et al. Patterns of lateral pelvic lymph node metastases and micrometastases for patients with lower rectal cancer. Eur J Surg Oncol 2007;33(4):463-467

59 Luna-Pérez P, Corral P, Labastida S, Rodríguez-Coria D, Delgado S. Inguinal lymph node metastases from rectal adenocarcinoma. J Surg Oncol 1999;70(3):177-180 
60 Schaap DP, Ogura A, Nederend J, et al. Prognostic implications of MRI-detected lateral nodal disease and extramural vascular invasion in rectal cancer. Br J Surg 2018;105(13):1844-1852 [Epub ahead of print]

61 Brown G, Richards CJ, Bourne MW, et al. Morphologic predictors of lymph node status in rectal cancer with use of high-spatial-resolution MR imaging with histopathologic comparison. Radiology 2003;227(2):371-377

62 Kim JH, Beets GL, Kim MJ, Kessels AG, Beets-Tan RG. Highresolution MR imaging for nodal staging in rectal cancer: are there any criteria in addition to the size? Eur J Radiol 2004;52(1):78-83

63 Matsuoka H, Nakamura A, Sugiyama M, Hachiya J, Atomi Y, Masaki T. MRI diagnosis of mesorectal lymph node metastasis in patients with rectal carcinoma. what is the optimal criterion? Anticancer Res 2004;24(6):4097-4101

64 Mir N, Sohaib SA, Collins D, Koh DM. Fusion of high b-value diffusion-weighted and T2-weighted MR images improves identification of lymph nodes in the pelvis. J Med Imaging Radiat Oncol 2010;54(4):358-364

65 Will O, Purkayastha S, Chan C, et al. Diagnostic precision of nanoparticle-enhanced MRI for lymph-node metastases: a meta-analysis. Lancet Oncol 2006;7(1):52-60

66 Bashir MR, Bhatti L, Marin D, Nelson RC. Emerging applications for Ferumoxytol as a contrast agent in MRI. J Magn Reson Imaging 2015;41(4):884-898

67 Lambregts DM, Beets GL, Maas M, et al. Accuracy of gadofosveset-enhanced MRI for nodal staging and restaging in rectal cancer. Ann Surg 2011;253(3):539-545

68 Lambregts DM, Heijnen LA, Maas M, et al. Gadofosvesetenhanced MRI for the assessment of rectal cancer lymph nodes: predictive criteria. Abdom Imaging 2013;38(4):720-727

69 Heijnen LA, Lambregts DM, Martens MH, et al. Performance of gadofosveset-enhanced MRI for staging rectal cancer nodes: can the initial promising results be reproduced? Eur Radiol 2014;24(2):371-379

70 Kantorová I, Lipská L, Bêlohlávek O, Visokai V, Trubaĉ M, Schneiderová M. Routine (18)F-FDG PET preoperative staging of colorectal cancer: comparison with conventional staging and its impact on treatment decision making. J Nucl Med 2003;44(11):1784-1788

71 Tsunoda Y, Ito M, Fujii H, Kuwano H, Saito N. Preoperative diagnosis of lymph node metastases of colorectal cancer by FDG-PET/CT. Jpn J Clin Oncol 2008;38(5):347-353

72 Koh DM, Brown G, Husband JE. Nodal staging in rectal cancer. Abdom Imaging 2006;31(6):652-659

73 Kim DJ, Kim JH, Ryu YH, Jeon TJ, Yu JS, Chung JJ. Nodal staging of rectal cancer: high-resolution pelvic MRI versus 18F-FDGPET/CT. J Comput Assist Tomogr 2011;35(5):531-534

74 Roh MS, Colangelo LH, O'Connell MJ, et al. Preoperative multimodality therapy improves disease-free survival in patients with carcinoma of the rectum: NSABP R-03. J Clin Oncol 2009;27(31):5124-5130

75 Patel UB, Blomqvist LK, Taylor F, et al. MRI after treatment of locally advanced rectal cancer: how to report tumor response-the MERCURY experience. AJR Am J Roentgenol 2012;199(4):W486-95

76 Chand M, Swift RI, Tekkis PP, Chau I, Brown G. Extramural venous invasion is a potential imaging predictive biomarker of neoadjuvant treatment in rectal cancer. $\mathrm{Br} \mathrm{J}$ Cancer 2014;110(1):19-25
77 Del Vescovo R, Trodella LE, Sansoni I, et al. MR imaging of rectal cancer before and after chemoradiation therapy. Radiol Med (Torino) 2012;117(7):1125-1138

78 Hussain SM, Outwater EK, Siegelman ES. Mucinous versus nonmucinous rectal carcinomas: differentiation with MR imaging. Radiology 1999;213(1):79-85

79 Petrillo A, Fusco R, Petrillo M, et al. Dynamic contrast enhanced MRI in locally advanced rectal cancer: value of time intensity curve visual inspection to assess neo-adjuvant therapy response. J. Physiol. Health 2014;110:255-267

80 Jung SH, Heo SH, Kim JW, et al. Predicting response to neoadjuvant chemoradiation therapy in locally advanced rectal cancer: diffusion-weighted 3 Tesla MR imaging. J Magn Reson Imaging 2012;35(1):110-116

81 van der Paardt MP, Zagers MB, Beets-Tan RG, Stoker J, Bipat S. Patients who undergo preoperative chemoradiotherapy for locally advanced rectal cancer restaged by using diagnostic MR imaging: a systematic review and meta-analysis. Radiology 2013;269(1):101-112

82 Enkhbaatar NE, Inoue S, Yamamuro $\mathrm{H}$, et al. MR Imaging with Apparent diffusion coefficient histogram analysis: evaluation of locally advanced rectal cancer after chemotherapy and radiation therapy. Radiology 2018;288(1):129-137

83 Sun YS, Zhang XP, Tang L, et al. Locally advanced rectal carcinoma treated with preoperative chemotherapy and radiation therapy: preliminary analysis of diffusion-weighted MR imaging for early detection of tumor histopathologic downstaging. Radiology 2010;254(1):170-178

84 DeVries AF, Kremser C, Hein PA, et al. Tumor microcirculation and diffusion predict therapy outcome for primary rectal carcinoma. Int J Radiat Oncol Biol Phys 2003;56(4):958-965

85 Quaia E, Gennari AG, Ricciardi MC, Ulcigrai V, Angileri R, Cova MA. Value of percent change in tumoral volume measured at T2-weighted and diffusion-weighted MRI to identify responders after neoadjuvant chemoradiation therapy in patients with locally advanced rectal carcinoma. J Magn Reson Imaging 2016;44(6):1415-1424

86 Birlik B, Obuz F, Elibol FD, et al. Diffusion-weighted MRI and MR-volumetry-in the evaluation of tumor response after preoperative chemoradiotherapy in patients with locally advanced rectal cancer. Magn Reson Imaging 2015;33(2):201-212

87 Horvat N, Veeraraghavan $H$, Khan M, et al. MR Imaging of rectal cancer: Radiomics analysis to assess treatment response after neoadjuvant therapy. Radiology 2018;287(3):833-843

88 Lambrecht M, Vandecaveye V, De Keyzer F, et al. Value of diffusion-weighted magnetic resonance imaging for prediction and early assessment of response to neoadjuvant radiochemotherapy in rectal cancer: preliminary results. Int J Radiat Oncol Biol Phys 2012;82(2):863-870

89 Lambregts DMJ, Yassien AB, Lahaye MJ, et al. Monitoring early changes in rectal tumor morphology and volume during 5 weeks of preoperative chemoradiotherapy - An evaluation with sequential MRIs. Radiother Oncol 2018;126(3):431-436

90 Dworak O, Keilholz L, Hoffmann A. Pathological features of rectal cancer after preoperative radiochemotherapy. Int J Colorectal Dis 1997;12(1):19-23

91 Aker M, Boone D, Chandramohan A, Sizer B, Motson R, Arulampalam T. Diagnostic accuracy of MRI in assessing tumor regression and identifying complete response in patients with locally advanced rectal cancer after neoadjuvant treatment. Abdom Radiol (NY) 2018;43:3213 